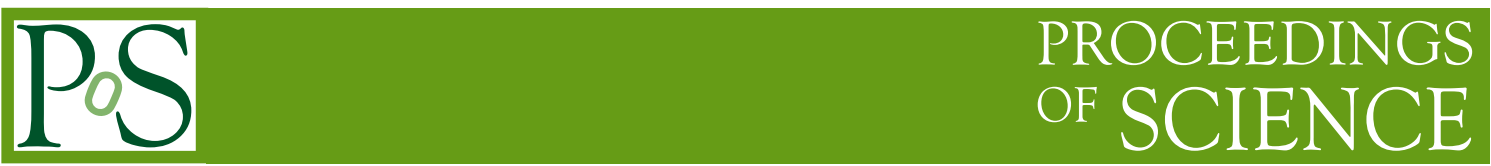

\title{
Aerogel RICH for Belle II
}

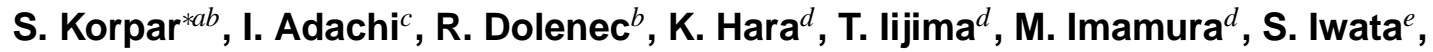 H. Kawai ${ }^{f}$, P. Križan ${ }^{g} b$, T. Kumita ${ }^{e}$, E. Kuroda ${ }^{e}$, S. Nishida ${ }^{c}$, S. Ogawa ${ }^{h}$, R. Pestotnik ${ }^{b}$, L. Šantelj ${ }^{b}$, A. Seljak $^{i b}$, S. Shiizuka ${ }^{d}$, T. Sumiyoshi $^{e}$, M. Tabata ${ }^{f j}$, S. Tagai ${ }^{h}$, R. Verheyden ${ }^{b}$}

${ }^{a}$ University of Maribor, Maribor, Slovenia

${ }^{b}$ Jožef Stefan Institute, Ljubljana, Slovenia

${ }^{c}$ High Energy Accelerator Research Organization (KEK), Tsukuba, Japan

${ }^{d}$ Nagoya University, Nagoya, Japan

${ }^{e}$ Tokyo Metropolitan University, Hachioji, Japan

${ }^{f}$ Chiba University, Chiba, Japan

${ }^{g}$ University of Ljubljana, Ljubljana, Slovenia

${ }^{h}$ Toho University, Funabashi, Japan

${ }^{i}$ Cosylab, Ljubljana, Slovenia

${ }^{j}$ Japan Aerospace Exploration Agency (JAXA), Sagamihara, Japan

E-mail: 'samo.korpar@ij.s.sì'

For the Belle-II experiment, a proximity focusing RICH counter with silica aerogel radiator will be employed as a new particle identifier in the forward endcap region. The detector makes use of a novel radiator concept, a multilayer radiator stack in a focusing configuration. The central element of the counter is a reliable single photon detector which can be operated at $1.5 \mathrm{~T}$, has a sufficiently fine granularity and compact size. A novel hybrid avalanche photo-detector (HAPD) has been developed with Hamamatsu to satisfy these conditions. We have studied the device on the bench, and in beam tests, and have shown that it can be successfully operated in a high axial magnetic field.

35th International Conference of High Energy Physics - ICHEP2010,

July 22-28, 2010

Paris France

* Speaker. 

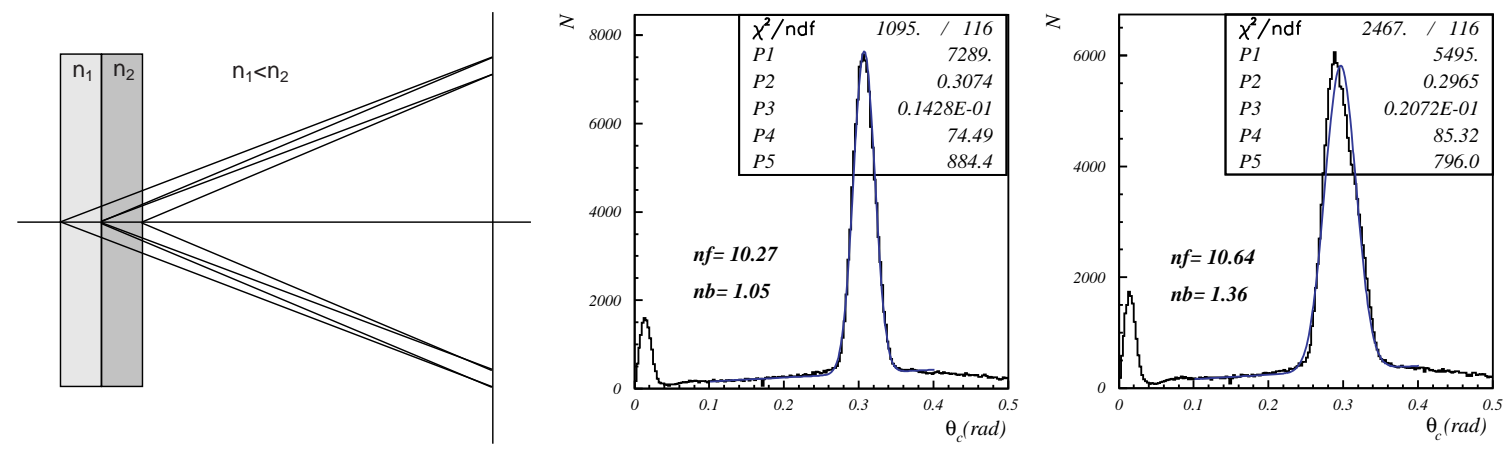

Figure 1: Proximity focusing RICH with a nonhomogeneous aerogel radiator in the focusing configuration(left); the accumulated distribution of Cherenkov photon hits depending on the corresponding Cherenkov angle for a $4 \mathrm{~cm}$ homogeneous radiator (right) and a focusing configuration with $n_{1}=1.046, n_{2}=1.056$ (center).

\section{Proximity focusing aerogel RICH}

For the next round of $B$ physics experiments at upgraded $e^{+} e^{-}$machines ('Super $B$ factories') with luminosities exceeding $10^{35} \mathrm{~cm}^{-2} \mathrm{~s}^{-1}$, considerable improvements of particle identification devices are envisaged in order to cope with higher rates and with more stringent requirements on separation capabilities for rare decay channels. For the endcap region of the upgraded Belle

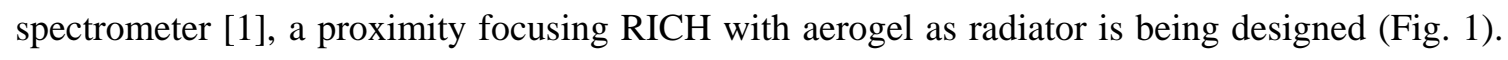
The counter should have a low threshold for pions, and should enable good separation of pions and kaons up to $4 \mathrm{GeV} / c$. Another benefit of such a counter would also be a reasonable $e / \mu / \pi$ separation at low momenta, which is of importance for the studies of rare $B \rightarrow K \ell \ell$ decays. The

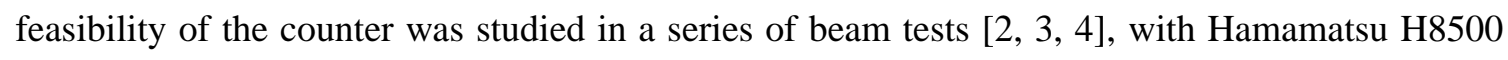

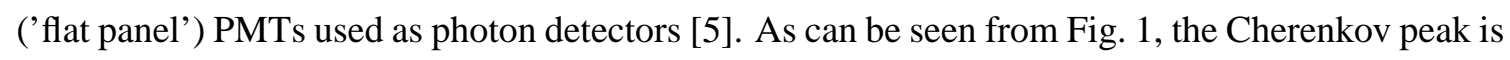
well pronounced above a small background, mainly coming from Cherenkov photons which were Rayleigh-scattered in the radiator.

The key issue in the performance of a RICH counter is to improve the Cherenkov angle resolution per track $\sigma_{\text {track }}=\sigma_{\theta} / \sqrt{N}$. With a longer radiator, the number of detected photons increases, but in a proximity focusing RICH the single photon resolution degrades because of the emission point uncertainty. As it turns out for the given geometry, the optimal thickness is around $20 \mathrm{~mm}\left[\begin{array}{l}2, \\ -1\end{array}\right]$. However, this limitation can be overcome in a proximity focusing RICH with a nonhomogeneous radiator $\left[\begin{array}{l}14 \\ 0\end{array}\right.$ aerogel radiator layers, one may achieve overlapping of the corresponding Cherenkov rings on the

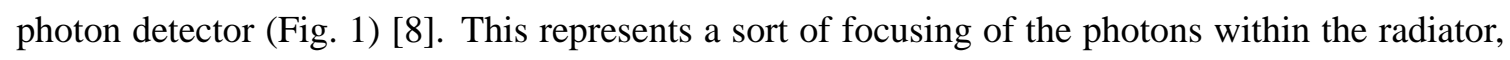
and eliminates or at least considerably reduces the spread due to emission point uncertainty. Note that such a tuning of refractive indices for individual layers is only possible with aerogel, which may be produced with any desired refractive index in the range 1.01-1.07 [19:-10 The dual radiator combination can readily be extended to more than two aerogel radiators. In this case, the indices of aerogel layers should gradually increase from the upstream to the downstream layer. In Fig. إin, we compare the data for two $4 \mathrm{~cm}$ thick radiators; one with aerogel tiles of equal refractive index $(\mathrm{n}=1.046)$, the other with the focusing arrangement $\left(n_{1}=1.046, n_{2}=1.056\right)$. The improvement is 

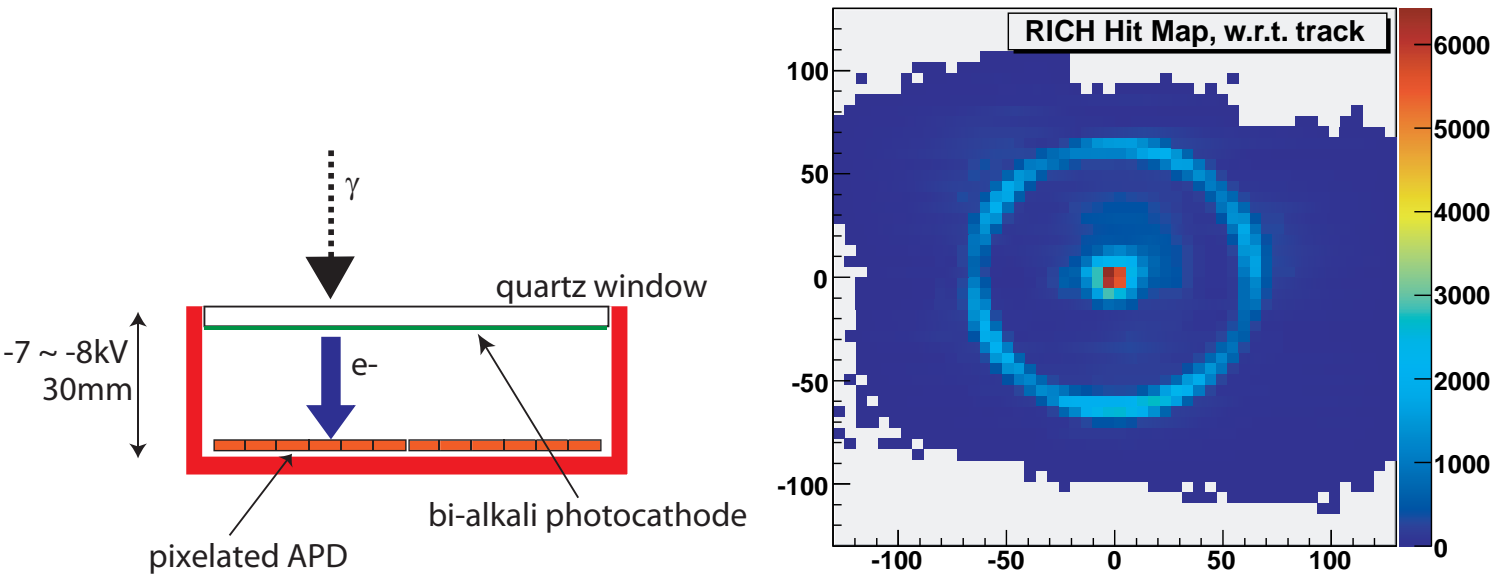

Figure 2: Multi-anode HAPD structure with 4.9 x $4.9 \mathrm{~mm}^{2}$ pads (left), and Cherenkov rings as recorded in a beam test at KEK (right) $\left[11_{1}^{1} 1_{b}^{1}, 1 \frac{1}{2}\right]$.

clearly visible. The single photon resolution $\sigma_{\theta}=14.3 \mathrm{mrad}$ for the dual radiator is considerably better than the corresponding value for the single refractive index radiator $\left(\sigma_{\theta}=20.7 \mathrm{mrad}\right)$, while the number of detected photons is the same in both cases.

\section{Photon detectors}

The present $R \& D$ efforts are oriented mainly toward the development of a highly efficient single photon detector which works in the high magnetic field (1.5 T) of the Belle spectrometer. The baseline photosensor, a novel type of hybrid photon detector of the proximity focusing type (Fig.'年), has been developed in a joint effort with the Hamamatsu company [1] counter, known as HAPD, photo-electrons are accelerated over a potential difference of $8 \mathrm{kV}$, and are detected in one of the four APD semiconductor sensors with 6x6 pads. The active area of the $72 \times 72 \mathrm{~mm}^{2}$ sensor is $65 \%$. Because the gain of the device (including the gain of $\approx 20$ in the APD) is rather low (about $\left.5 \cdot 10^{4}\right)$, while its capacitance is quite high $(80 \mathrm{pF})$, it is read-out by dedicated custom-made high gain and low noise electronics. In a test beam at KEK, Cherenkov rings were recorded with an array of six such sensors, as shown in Fig. $\bar{z}_{\text {. }}$. The counter was also successfully operated in a magnetic field of $1.5 \mathrm{~T}$.

As a backup option, the Photonis Planacon 85011 micro-channel plate (MCP) PMT [1] device with an $8 \times 8$ array of $6 \mathrm{~mm} \times 6 \mathrm{~mm}$ large pads, has been shown to perform very well as a detector of Cherenkov photons in a RICH counter [i] cross-talk and the excellent timing properties of the tube were further investigated. It was also shown that with such a photo-sensor, the proximity focusing RICH counter could also be used as a time-of-flight counter using Cherenkov photons emitted in the PMT window [1] ${ }_{-1}^{1} \bar{\sigma}_{1}^{\prime}$. As the third option, Geiger mode APDs, also known as silicon photomultipliers, equipped with dedicated light concentrators have been tested on the bench and in a test beam. They were shown to be an excellent

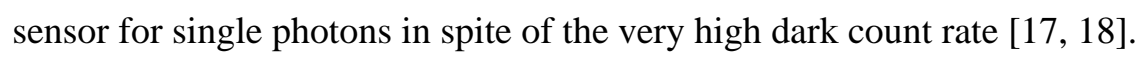




\section{Conclusions}

For the proximity focusing RICH counter of the Belle-II experiment, we have developed a novel radiator concept, a multilayer radiator stack in a focusing configuration. We have also studied three different photon detectors, capable of operation in a high magnetic field, with a sufficiently fine granularity and compact size. As the baseline option we have chosen a novel hybrid avalanche photo-detector (HAPD) that has been developed in collaboration with Hamamatsu. The device showed excellent performance on the bench and in test beams, and could be successfully operated in an axial magnetic field of $1.5 \mathrm{~T}$.

\section{References}

[1] T. Abe et al., "Belle II Technical Design Report," arXiv:1011.0352 [physics.ins-det].

[2] T. Matsumoto, S. Korpar et al., Nucl. Instr. and Meth. A 521 (2004) 367.

[3] P. Križan et al., Nucl. Instr. and Meth. A 533 (2005) 58.

[4] T. Iijima, S. Korpar et al., Nucl. Instr. and Meth. A 548 (2005) 383.

[5] H8500 data sheet, Hamamatsu Photonics K.K.

[6] P. Križan, Super B Factory Workshop, 19-22 Jan 2004, Honolulu, Hawaii, http://www.phys.hawaii.edu/superb04.

[7] A. Danilyuk et al., Nucl. Instr. and Meth. A 533 (2005) 70.

[8] P. Križan, S. Korpar, T. Iijima, Nucl. Instr. and Meth. A 565 (2006) 457.

[9] I. Adachi et al., Nucl. Instr. and Meth. A 533 (2005) 146.

[10] A.Yu. Barnyakov et al., 595 (2008) 100.

[11] S. Nishida et al., Nucl. Instr. and Meth. A 595 (2008) 150.

[12] I. Adachi et al., Nucl. Instr. and Meth. A 623 (2008) 285; I. Adachi et al., Nucl. Instr. and Meth. (2010), doi:10.1016/j.nima.2010.09.054.

[13] BURLE 85011 data sheet: http//www.burle.com/cgi-bin/byteserver.pl/pdf/85011-501.pdf

[14] P. Križan et al., Nucl. Instr. and Meth. A 567 (2006) 124.

[15] S. Korpar et al., Nucl. Instrum. Meth. A 595 (2008) 169; S. Korpar et al., Nucl. Instrum. Meth. A (2010), doi:10.1016/j.nima.2010.10.148.

[16] S. Korpar et al., Nucl. Instr. and Meth. A 572 (2007) 423.

[17] S. Korpar et al., Nucl. Instrum. Meth. A 594 (2008) 13.

[18] S. Korpar et al., Nucl. Instr. and Meth. A 613 (2010) 195. 